\title{
Elimination of a Carcinogenic Anionic Dye Congo Red from Water Using Hydrogels Based on Chitosan, Acrylamide and Graphene Oxide
}

\section{Amtul Jamil Sami*, Yasha Nazir Butt and Sitara Nasar}

Institute of Biochemistry and Biotechnology, University of the Punjab, Lahore, 54590, Pakistan

*Corresponding author: Sami AJ, Institute of Biochemistry and Biotechnology, University of the Punjab, Lahore, 54590, Pakistan, Tel: 92-42-99231596; E-mail: amtuljamilsami@yahoo.com

Received date: July 11, 2018; Accepted date: July 24, 2018; Published date: July 31, 2018

Copyright: (C) 2018 Sami AJ, et al. This is an open-access article distributed under the terms of the Creative Commons Attribution License, which permits unrestricted use, distribution and reproduction in any medium, provided the original author and source are credited.

\begin{abstract}
Industrial effluents discharged from textile mills and tanneries contain carcinogenic dyes which cause environmental and health issues. Harmful dyes such as Congo red can be removed from water using hydrogels which were developed and characterized to study efficient dye removal through adsorption mechanism. Chitosan polyacrylamide composite hydrogels showed promising results of $79 \%$ dye removal with maximum adsorption of 2.1 $\times 10^{-4} \mathrm{~mol} \mathrm{~g}^{-1}$. At $\mathrm{pH} \mathrm{7,} \mathrm{these} \mathrm{hydrogels} \mathrm{followed} \mathrm{case-Il} \mathrm{diffusion} \mathrm{mechanism} \mathrm{and} \mathrm{showed} 185.3 \%$ swelling. Similarly, novel Nano composite hydrogels containing graphene oxide as additional component extracted from battery cells had swelling percentage of $227 \%$. At the same $\mathrm{pH}$, these hydrogels had $94.7 \%$ dye removal with adsorption of $3.5 \times 10^{-4} \mathrm{~mol} \mathrm{~g}^{-1}$. About $200 \mathrm{mg}$ of Congo red was removed by $1 \mathrm{~g}$ of chitosan present in the hydrogels. SEM analysis and IR spectra confirmed the adsorption of Congo red dye onto hydrogels and hydrogels demonstrating pores size of $80 \mu \mathrm{m}$ masked by the dye particles. Anti-microbial activity was also confirmed for both hydrogels with clear zone of inhibition against $E$. coli JM 109. The adsorption experimental data agreed well with pseudo second order reaction kinetics and Langmuir isotherm with regression values of $97 \%$ and $99 \%$. The applicability of these hydrogels towards adsorption of commercial dyes was also determined by using wastewater sample obtained from local fabric-dyeing vendor where the results highlighted $85 \%$ and $85 \%$ adsorption.
\end{abstract}

Keywords: Adsorption; Congo red; Hydrogels; Chitosan; Decontamination; Kinetics

\section{Abbreviations:}

$\mathrm{C}_{\mathrm{o}}$ : Initial concentration of dye $\left(\mathrm{mg} \mathrm{L}^{-1}\right) ; \mathrm{C}_{\mathrm{e}}$ : Concentration of dye at equilibrium in $\left(\mathrm{mgL}^{-1}\right)$; $\mathrm{C}_{\mathrm{t}}$ : Final concentration of dye in $\left(\mathrm{mgL}^{-1}\right)$; $\mathrm{H}$ : Initial weight of Hydrogel $(\mathrm{g}) ; \mathrm{H}^{\mathrm{o}}$ : Final weight of Hydrogel (g); K: Characteristic constant; $\mathrm{K}_{\mathrm{f}}$. Freundlich adsorption constant $\left(\mathrm{mg} \mathrm{g}^{-1}\right)$; $\mathrm{k}_{1}$ : Rate constant for Pseudo 1st order reaction $\left(\mathrm{min}^{-1}\right) ; \mathrm{k}_{2}$ : Rate constant for Pseudo 2nd order reaction $\left(\mathrm{mg} \mathrm{g}^{-1} \mathrm{~min}^{-1}\right)$; $\mathrm{m}$ : Mass of adsorbent $(\mathrm{g}) ; \mathrm{M}_{\mathrm{t}}$ : Amount of water adsorbed at time $\mathrm{t} ; \mathrm{M}_{\infty}$ : Amount of water adsorbed at equilibrium; $\mathrm{Q}_{\mathrm{e}}$ : Amount of dye per gram of adsorbent $\left(\mathrm{mg} \mathrm{g}^{-1}\right)$; $\mathrm{Q}_{\mathrm{t}}$ : Amount of dye per gram of adsorbent $\left(\mathrm{mg} \mathrm{g}^{-1}\right)$; $\mathrm{t}$ : time (min); $\mathrm{t}_{0.5}$ : Square root value of time (min 0.5); V: Volume of the solution $(\mathrm{L}) ; \mathrm{W}_{\mathrm{t}}$ : Initial mass of adsorbent $(\mathrm{g})$; $\mathrm{W}_{\mathrm{o}}$ : Final mass of adsorbent $(\mathrm{g})$.

\section{Introduction}

Recently, the removal of environmental pollutants from natural water bodies and wastewater has gained tremendous importance due to rapid growth of population, urbanization, industrialization, and agricultural impacts on the environment. These sectors give way to the discharge of harmful waste material such as synthetic dyes directly or indirectly into the water bodies [1]. Most of these organic dyes released from textile, printing, and leather industries are mutagenic, carcinogenic, and toxicogenic and cause severe health disorders such as cancer, skin problems, heart diseases, jaundice, mutations, and allergies on becoming part of food chain in ecological system [2]. The discharge of bulk quantities of brilliant color dyes into natural waters has led to aesthetical issues and has caused great harm to the ecosystem. Azodyes compounds are coloring agents often found in the water bodies beyond permissible limits as set by stringent regulations [3]. Congo red dye is an anionic azodye which metabolizes in body to produce carcinogenic compounds such as benzidine. Thus, purification of water bodies is very important due to the complete irreversibility of dyes towards biodegradative processes in nature [4].

Today, there is an increased demand of novel polymers in the industrial sectors for the effective removal and separation of dyes. Different chemical, physical, biological, and physico-chemical technologies such as filtration, reverse osmosis, solvent extraction, distillation, flocculation, and coagulation are being used to remove harmful pollutants from aqueous bodies [5]. Similarly adsorption is an effective separation technique, in which molecules are held on the surface of the material and is popular due to cost-effectiveness, simplicity, efficacy, and non-reactiveness towards toxic agents. The use of synthetic hydrogels for decontamination of wastewater through adsorption is an efficient technique. It makes use of hydrophilic polymers that have ability to adsorb water without being dissolved [6]. Hydrogels or 'smart' and 'intelligent' gels are 3-D polymeric crosslinked network based hydrogels with capability of holding high amount of liquid which can either be water, biological fluid, or any other solution [7]. A number of hybrid hydrogels are now being designed for the removal of cationic and anionic dyes, metal ions, and radionuclides. Among many biopolymers of great significance, chitosan is obtained from shrimps and insects. It is extracted through deacetylation of chitin [8]. When combined with acrylamide monomers together with initiators and crosslinkers, chitosan embeds in the polyacrylamide network of hydrogels where it provides hydrogel 
with antimicrobial activity and reactive hydrophilic functional groups for greater water retention and high degree of swellings $[9,10]$.

Hydrogels with higher degree of hydration has greater adsorption capacity which holds strong basis for wastewater treatment [11-13]. Surface adsorption using hydrogels is one of the most cost-effective and efficient ways to remove harmful and carcinogenic dyes from wastewater. The mechanism of dye removal using chitosan based polymeric hydrogels with embedded graphene oxide involve different interactions such as Van der Waals forces, electrostatic forces, and ionic interaction [14-16]. The polymers offer a large number of free reactive functional groups available for dye molecules for binding whereas graphene oxide provides hydrogels with strength, stability, and intactness [17].

This study discusses the production and characterization of effective synthetic Chitosan (CS) polyacrylamide (PAM) hydrogels with and without graphene oxide for efficient removal of carcinogenic Congo red (CR) dye from aqueous solution and commercial wastewater sample. It also describes the mechanism and kinetics of adsorption of Congo red, swelling kinetics at different $\mathrm{pH}$ values, water retention values (WRVs), and equilibrium studies. Furthermore, this study explores the characteristic properties of test hydrogels and control hydrogels with and without adsorbed Congo red dye (CR) through FTIR (Fourier Transform Infrared Spectroscopy) and SEM (Scanning Electron Microscope) analysis. Finally, it also discusses the antimicrobial activity of hydrogels for the inhibition of bacterial growth against decomposition of hydrogel when placed in water bodies. Recently, the removal of environmental pollutants from natural water bodies and wastewater has gained tremendous importance due to rapid growth of population, urbanization, industrialization, and agricultural impacts on the environment. These sectors give way to the discharge of harmful waste material such as synthetic dyes directly or indirectly into the water bodies [18]. Most of these organic dyes released from textile, printing, and leather industries are mutagenic, carcinogenic, and toxicogenic and cause severe health disorders such as cancer, skin problems, heart diseases, jaundice, mutations, and allergies on becoming part of food chain in ecological system [19]. The discharge of bulk quantities of brilliant color dyes into natural waters has led to aesthetical issues and has caused great harm to the ecosystem. Azodyes compounds are coloring agents often found in the water bodies beyond permissible limits as set by stringent regulations [20]. Congo red dye is an anionic azodye which metabolizes in body to produce carcinogenic compounds such as benzidine. Thus, purification of water bodies is very important due to the complete irreversibility of dyes towards biodegradative processes in nature $[21,22]$.

Today, there is an increased demand of novel polymers in the industrial sectors for the effective removal and separation of dyes. Different chemical, physical, biological, and physico-chemical technologies such as filtration, reverse osmosis, solvent extraction, distillation, flocculation, and coagulation are being used to remove harmful pollutants from aqueous bodies [6]. Similarly adsorption is an effective separation technique, in which molecules are held on the surface of the material and is popular due to cost-effectiveness, simplicity, efficacy, and non-reactiveness towards toxic agents. The use of synthetic hydrogels for decontamination of wastewater through adsorption is an efficient technique. It makes use of hydrophilic polymers that have ability to adsorb water without being dissolved [4]. Hydrogels or 'smart' and 'intelligent' gels are 3-D polymeric crosslinked network based hydrogels with capability of holding high amount of liquid which can either be water, biological fluid, or any other solution [9]. A number of hybrid hydrogels are now being designed for the removal of cationic and anionic dyes, metal ions, and radionuclides. Among many biopolymers of great significance, chitosan is obtained from shrimps and insects. It is extracted through deacetylation of chitin [13]. When combined with acrylamide monomers together with initiators and crosslinkers, chitosan embeds in the polyacrylamide network of hydrogels where it provides hydrogel with antimicrobial activity and reactive hydrophilic functional groups for greater water retention and high degree of swellings [15].

Hydrogels with higher degree of hydration has greater adsorption capacity which holds strong basis for wastewater treatment $[8,9]$. Surface adsorption using hydrogels is one of the most cost-effective and efficient ways to remove harmful and carcinogenic dyes from wastewater. The mechanism of dye removal using chitosan based polymeric hydrogels with embedded graphene oxide involve different interactions such as Van der Waals forces, electrostatic forces, and ionic interaction [3]. The polymers offer a large number of free reactive functional groups available for dye molecules for binding whereas graphene oxide provides hydrogels with strength, stability, and intactness [23].

This study discusses the production and characterization of effective synthetic Chitosan (CS) polyacrylamide (PAM) hydrogels with and without graphene oxide for efficient removal of carcinogenic Congo red (CR) dye from aqueous solution and commercial wastewater sample. It also describes the mechanism and kinetics of adsorption of Congo red, swelling kinetics at different $\mathrm{pH}$ values, water retention values (WRVs), and equilibrium studies. Furthermore, this study explores the characteristic properties of test hydrogels and control hydrogels with and without adsorbed Congo red dye (CR) through FTIR (Fourier Transform Infrared Spectroscopy) and SEM (Scanning Electron Microscope) analysis. Finally, it also discusses the antimicrobial activity of hydrogels for the inhibition of bacterial growth against decomposition of hydrogel when placed in water bodies.

\section{Materials and Methods}

\section{Materials}

Analytical grade chemicals' including commercial chitosan (DDA-75, Mol. Wt: 17100) was purchased from biology biotechnologies und logistik gmbh, Germany. Congo red dye and ammonium per sulfate (APS) were obtained from Sigma Aldrich. TEMED (Tetramethylethylenediamine) was purchased from UniCHEM, Bis-acrylamide from Fluka BioChemika, and Acrylamide from Acros Organics. Glycerol was purchased from Omicron Sciences Limited. Absolute ethanol and potassium hydroxide chemicals were obtained from Analar, BDH Company. Recycled source of graphite for the production of graphene oxide was obtained from used batteries.

\section{Fabrication}

Preparation of hydrogels: The first set of polymeric hydrogels was prepared by mixing $1.2 \mathrm{wt} \%$ Chitosan, $4 \mathrm{wt} \%$ Acrylamide, $0.8 \mathrm{wt} \%$ Bisacrylamide, $0.002 \mathrm{wt} \%$ TEMED, and $0.02 \mathrm{wt} \%$ APS, and $4 \mathrm{ml}$ glycerol; whereas, the second set of hydrogels was prepared by additionally adding $0.0032 \mathrm{wt} \%$ graphene oxide (GO) prepared from graphite in batteries through modified Hummer's method [24]. These components were mixed in $20 \mathrm{ml}$ of $2 \mathrm{wt} \%$ acetic acid solution and loaded into the moulds for overnight polymerization at $55^{\circ} \mathrm{C}$. Fixation solution comprising of $1 \mathrm{~g}$ potassium hydroxide $(\mathrm{KOH}), 4 \mathrm{ml}$ glycerol, and 96 
$\mathrm{ml}$ absolute ethanol was mixed well and used for better flexibility, neutralization, and elasticity of the hydrogels [25]. All the experimental studies were carried out at room temperature $25^{\circ} \mathrm{C}$ and at $\mathrm{pH}$ 7.0. Figure 1 shows the crosslinking network of the hydrogels.

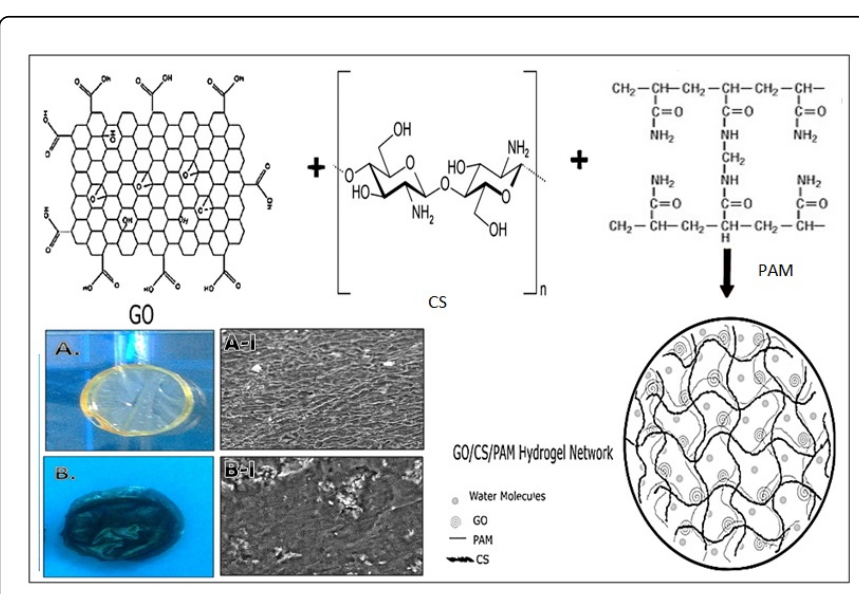

Figure 1: Cs-PAM hydrogel (A) CS-PAM-GO (B) with crosslinking network (A-I and A-II).

Swelling and kinetics: The swelling property of hydrogels is determined by effective imbibition of water molecules which supports high adsorption of dye molecules on hydrogels. Swelling of hydrogels depends on the nature, availability of functional groups, degree of cross-linking network of hydrogel, and $\mathrm{pH}$ changes in aqueous solutions. The swelling ability at variable $\mathrm{pH}$ was measured by immersing disks of hydrogels in the solutions including $\mathrm{pH}(2,7,10$, and 12). Hydrogel swelling data for three consecutive days was recorded by measuring the weight of swollen samples to further determine the swelling kinetics of hydrogels. Excess water on the surface of hydrogels was removed by slightly tapping the surface of hydrogel with the piece of filter paper. The degree of swelling at a particular time $t$, at which equilibrium was achieved, was monitored and further swelling ratio with respect to $\mathrm{pH}$ was calculated [26].

Swelling ratio (SR) of hydrogel

$\mathrm{SRx}=\mathrm{H} / \mathrm{H}^{\circ}(1)$

Where,

$\mathrm{H}=$ Final Weight;

$\mathrm{H}^{\circ}=$ Initial Weight.

Mechanism for water sorption through hydrogels was calculated to determine the mechanism through an empirical equation is mentioned below:

$$
F+\frac{M t}{M_{\infty}}=k t^{n}
$$

Where $\mathrm{F}$ is the swelling fractional constant, $\mathrm{Mt}$ is the amount of water intake by the hydrogel at a certain time $\mathrm{t} M \infty$ is the amount of water adsorbed at equilibrium, $\mathrm{k}$ represents the characteristic constant of hydrogel and $\mathrm{n}$ signifies characteristic exponent which describes the mechanism of water transport followed by the hydrogel.

Many hydrogel swelling models follow Fick's law of diffusion. According to the diffusion mechanism, in Fick's law, the net flux of atoms is in opposite direction to the concentration gradient. For hydrogels it can be considered as the mass transport of analyte or solvent molecules through convection mechanism. To determine the type of diffusion, the diffusion exponent value of $n$ helps to decide the transport mechanism of hydrogel. When $n$ is equal to 0.5 the hydrogel follows Fickian diffusion (Case I). When $\mathrm{n}$ is greater than 0.5 but less than 1 then hydrogel observes non-Fickian or anomalous diffusion. Lastly, if $\mathrm{n}$ is equal or greater than 1 then it follows Case II or super Case II diffusion mechanism. Fickian diffusion has least amount of water uptake and it is diffusion controlled while non-Fickian has greater water uptake [3].

IR spectra of hydrogels: The Fourier transform infrared (FTIR) spectra of hydrogels were analyzed using IR Prestige-21 Shimadzu spectrometer of resolution $4 \mathrm{~cm}^{-1}$ and effective range of 500 to 4000 $\mathrm{cm}^{-1}$. Each IR spectra was obtained through this spectrometer with the average of 70 scans. The IR spectra of the composite hydrogels, before and after dye removal were analyzed.

Scanning electron microscope analysis: Scanning electron microscopy (SEM) analysis was conducted using Hitachi s-3700 with magnification range of 500X to $300000 \mathrm{X}$. The characteristic network based photographs were obtained through this powerful technique with this high order of magnification. Characteristics including surface topography, cross-linkages and channels, composition, and pore size of the specimen were determined. Images were taken at PITMEAC within the range of $500 \mathrm{X}$ to $300000 \mathrm{X}$ at $15.0 \mathrm{kV}$ and noticeable images were selected.

Anti-microbial activity: Hydrogels are prone to microbial interference when used in purification process therefore it is necessary to add biocidal-agents such as chitosan and graphene oxide to the hydrogel. The anti-microbial activity of hydrogels was determined by preparing L.B. agar plates containing phenyl red indicator. $100 \mathrm{ml} \mathrm{L.B}$ agar media was prepared by mixing $0.5 \mathrm{~g}$ yeast extract, $1 \mathrm{~g} \mathrm{NaCl}$, and 1 $\mathrm{g}$ Tryptone. The $\mathrm{pH}$ was set to 7.4 and $1.5 \mathrm{~g}$ agar and $0.1 \mathrm{~g}$ of phenyl red was added to the solution which was further autoclaved. The autoclaved L.B agar medium was poured in sterile petri plates under sterile conditions in Laminar Flow (LF) Hood. $150 \mu$ of Escherichia coli JM 109 strain was spread onto the two plates including one for hydrogel samples and the other for positive control. The third plate was negative control without any strain. In sample plate, two hydrogels (with and without graphene oxide) were placed and slightly pressed for intactness. All three plates were covered with parafilm and placed inside oven at $37^{\circ} \mathrm{C}$ overnight growth. Zone of inhibition around hydrogels in test plates was determined the following day.

Folding endurance: Folding endurance of hydrogel was calculated by first repeatedly folding the hydrogels of $20 \mathrm{~cm}^{2}$. Hydrogels were folded at the same place and number of folds was counted until hydrogel was broken. This produced value of folding endurance of hydrogels which is to the $\log 10$ as given in equation 3 .

\section{$\mathrm{Fd}=\log 10 \mathrm{~d}(3)$}

Here, Fd indicates folding endurance, $\mathrm{d}$ is the number of double folds which are counted until hydrogel breaks.

Adsorption studies: The effective adsorption of Congo red dye onto hydrogels was determined by first preparing $0.1 \%$ stock solution of Congo red dye. $1000 \mathrm{mg}$ of congo red dye was dissolved in $1000 \mathrm{ml}$ of distilled water to prepare solution of $1000 \mathrm{mg} / \mathrm{L}$. Adsorption experiment was conducted by preparing working solution of $5 \mathrm{x}$ concentration from the stock solution of $0.1 \%$ Congo red dye and the $\mathrm{pH} 7$ of these solutions were set using $0.1 \mathrm{~N} \mathrm{NaOH}$ and $0.1 \mathrm{~N} \mathrm{HCl}$ 
respectively. Each adsorbent hydrogel disk used in experimental analysis had diameter of $2.5 \mathrm{~cm}$ and weighed $0.14 \mathrm{~g}$ each. Each hydrogel was immersed in $20 \mathrm{ml}$ of concentration $5 \mathrm{x}$ of Congo red dye solution separately in $100 \mathrm{ml}$ conical flasks followed by shaking at 370 rpm on an orbital shaker for 120 minutes at room temperature $25^{\circ} \mathrm{C}$. The respective absorbance for each of the solution was determined using UV/VIS spectrophotometer at wavelength of $540 \mathrm{~nm}$ after every half an hour based interval [27].

Adsorption kinetics based calculations were done to determine the amount of dye adsorbed by the hydrogel at a certain time " $t$ ". There is a mass balance equation which helps to determine the amount which is as follows:

$$
Q t=\frac{\left(C_{\mathrm{o}}-C_{t}\right) V}{m}
$$

Qt is the amount of dye which gets adsorbed at certain time;

$\mathrm{C}_{\mathrm{o}}$ is the initial concentration of dye and $\mathrm{C}_{\mathrm{t}}$ is concentration of dye at time " $\mathrm{t}$ ";

$\mathrm{V}$ is the volume of solution an $\mathrm{m}$ is the mass of hydrogel in grams.

Further, percentage adsorption was calculated through the following equation [6]:

$$
\% \text { Adsorption }=\frac{C_{\circ}-C_{t}}{C_{\circ}} \times 100 \text {. }
$$

Since change in concentration of solution containing dyes have a great impact on adsorption, experimental studies were carried out to confirm the direct proportion relationship between dye molecules of solute and the adsorptive reactions. The mechanism of adsorption was determined by examining the transient behavior of dye molecules in adsorption process analyzed through pseudo first order reaction and pseudo second order reaction.

Pseudo first order of reaction was determined by the following equation [3];

$$
\log \left(q_{e}-q_{t}\right)=\log q e-\frac{k 1}{2.303} t
$$

Where, qe and qt ( $\mathrm{mg} / \mathrm{g})$ are the amounts of dye that are adsorbed at equilibrium and at time " $\mathrm{t}$ ". The graph between values log (qe-qt) vs $t$ was plotted. Pseudo second order of reaction was calculated by the following equation.

$$
\frac{t}{q t}=\frac{1}{k 2 q e 2}+\frac{1}{q e} t
$$

A plot between $t$ /qt vs $t$ was also drawn to determine slope and intercept for $\mathrm{k}$ and qe.

The adsorption isotherms were also selected to determine the interaction of adsorbent. Langmuir isotherm of linear form was obtained by plotting a graph of Ce/qe Vs Ce and was calculated through the following equation:

$$
\frac{C_{e}}{q_{e}}=\left(\frac{1}{k a q m}\right)+\frac{C_{e}}{q m} .
$$

Where $\mathrm{qm}(\mathrm{mg} / \mathrm{g})$ is the maximum amount of adsorption and $\mathrm{Ka}$ is the Langmuir constant.
Freundlich isotherms described the adsorption on heterogeneous adsorbent surfaces. It was obtained by plotting a graph between $\ln \mathrm{Ce}$ and $\ln$ qe and was calculated through the equation below:

$$
\ln q e=\operatorname{ink}_{f}+\frac{1}{n}+\ln C_{e}
$$

Where $\mathrm{q}_{\mathrm{e}}(\mathrm{mg} / \mathrm{g})$ refers to the amount of adsorbate on adsorbent at equilibrium and $\mathrm{Ce}$ is the concentration of adsorbate present in the solution at equilibrium. Kf and $\mathrm{n}$ represent isotherm constants for extent of adsorption.

\section{Application}

Dye adsorption using commercial sample: The industrial application of hydrogels was determined to check effectiveness of hydrogels towards adsorption of harmful dyes from wastewater. Commercial wastewater samples were collected from textile dyeing shop of a local vendor to qualitatively check the feasibility of dye removal by the CS-PAM and CS-PAM-GO hydrogels. Both the hydrogels were immersed in three different color containing flasks including red, yellow, and green colored dyes. The flasks were subjected to overnight shaking and absorption spectrum was recorded accordingly.

\section{Results}

\section{Characterization of hydrogels}

Swelling analysis and kinetic studies: Swelling is a basic principle for adsorption, where hydrogel with optimum swelling percentage gives favorable dye adsorption results. To monitor the optimum $\mathrm{pH}$ for greater adsorption of CR dye from the solution, swelling analysis of hydrogels was performed at $\mathrm{pH}$ 7. The water uptake by hydrogels increased with time until it reached equilibrium where water was no further gained by the hydrogels. The swelling percentage of CS-PAM hydrogel was calculated to be $185.3 \%$ while CS-PAM-GO showed higher swelling percentage of $227 \%$ respectively as shown in Figure 2A. The possibility behind enhanced percentage swelling in CS-PAM-GO hydrogels might be due to synergetic inter-molecular interactions present between polymeric networks and GO sheets to hold water leading to enhancement of swelling capacities. The nature of these hydrogels demonstrated hydrophilicity due to the presence of major hydrophilic groups in chitosan (DDA-75, Mol. Wt: 17100) including amine $\left(\mathrm{NH}_{2}\right)$ and hydroxyl $(-\mathrm{OH})$ groups. Due to hydrophilicity, these hydrogels responded to hydration. Also, greater number of hydrophilic groups present in the surface of GO sheets improved diffusion of water in the polymeric networks.

Swelling based kinetics was calculated through calculations using Equations 1 and 2. Graphs of $\log M_{t} / M_{\infty}$ Vs $t$ (h) was plotted accordingly as shown in Figures 2B and 3A. 

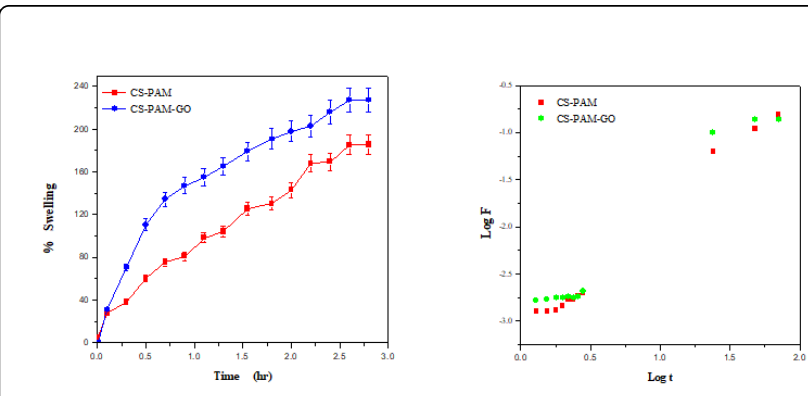

Figure 2: (2A) Percentage swelling of CS-PAM and CS-PAM-GO hydrogels at $\mathrm{pH} 7$ (left); (2B) $\log \mathrm{Mt} / \mathrm{M} \infty$ as a function of time for Swelling kinetics of CS-PAM and CS-PAM-GO hydrogels (right).

The constants $\mathrm{k}$ and $\mathrm{n}$ were acquired from intercepts and slopes from the graphs based on the experimental data as shown in Table 1. The values of $\mathrm{n}$ were assessed, and swelling mechanisms were determined for both types of hydrogels with and without graphene oxide.
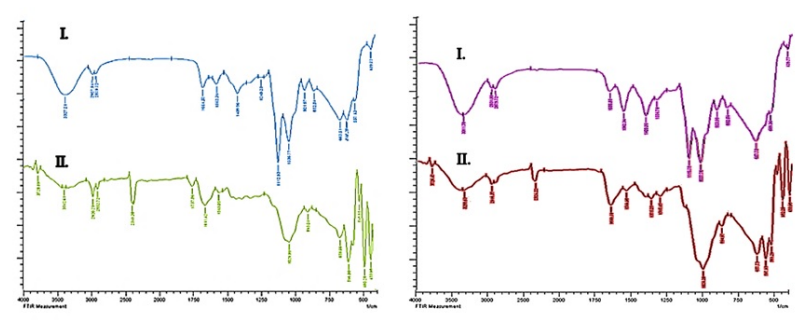

Figure 3: (3A) FTIR spectra of CS-PAM hydrogel without Congo red dye (I) with Congo red dye (II) (left) (3B) FTIR spectra of GOCS-PAM hydrogel without Congo red dye (I) with Congo red dye (II) (right).

\begin{tabular}{|l|l|l|l|l|l|}
\hline Hydrogel & $\mathbf{p H}$ & $\mathbf{n}$ slope & $\mathbf{k}$ intercept & $\mathbf{R 2}$ Regression (\%) & Mechanism Type \\
\hline CS-PAM & 7 & 1.3 & -3.2 & 98 & Case II Diffusion \\
\hline CS-PAM-GO & 7 & 1.4 & -3.3 & 98 & Case II Diffusion \\
\hline
\end{tabular}

Table 1: Swelling Kinetics.

Swelling mechanism followed by the hydrogels was determined Case-II diffusion mechanism with $\mathrm{n}$ values within the range of 1.3 and 1.4. This showed that hydrogels had greater water uptake.

At $\mathrm{pH} \mathrm{7,} \mathrm{the} \mathrm{amino} \mathrm{group} \mathrm{of} \mathrm{chitosan} \mathrm{offered} \mathrm{hydrophilicity}$ therefore increasing water uptake. The regression values with the range of 0.98 for these hydrogels were adequate. The degree of swelling is to a quite extent influenced by the presence of polar groups. The availability of polar groups is higher when there is less crosslinking within the polymeric composite hydrogels. Similar studies have been done by [21]. Case II transport of water and dye molecules attributes to higher hydrophilicity offered by the hydrogel. These diffusion behaviors of hydrogels describe comparable rates between relaxation and diffusion (Rdiff $\approx$ Rrelax).

FTIR analysis: The IR spectra of CS-PAM hydrogels and CS-PAMGO hydrogels were analyzed before and after Congo red dye adsorption, in order to confirm the binding of adsorbent with the adsorbate (Figures $3 \mathrm{~A}$ and $3 \mathrm{~B}$ ). Chitosan absorptions were observed at $3352.3 \mathrm{~cm}^{-1}$ and $3344.6 \mathrm{~cm}^{-1}$ for $-\mathrm{NH}_{2}$ and $-\mathrm{OH}$ groups with broad band peaks based stretching vibrations of both inter and intra molecular hydrogen bonds. Peaks of amide included $\mathrm{C}=\mathrm{O}$ stretching vibrations of chitosan located at $1656.8 \mathrm{~cm}^{-1}$ and $1649.1 \mathrm{~cm}^{-1}, \mathrm{~N}-\mathrm{H}$ vibrations located at $1543.1 \mathrm{~cm}^{-1}$ and $1589.0 \mathrm{~cm}^{-1}$, whereas; C-N stretching and bending vibrations were observed through peaks located at $1329.0 \mathrm{~cm}^{-1}$ and $1316.5 \mathrm{~cm}^{-1}$ [16].

There were several bands exhibited by CS-PAM hydrogels and CSPAM-GO hydrogels due to the presence of polyacrylamide including $\mathrm{N}-\mathrm{H}$ stretching vibrations at $3342.6 \mathrm{~cm}^{-1}$ and $3286.3 \mathrm{~cm}^{-1}$. There were $\mathrm{C}=\mathrm{O}$ stretching vibrations of polyacrylamide located at $1601.0 \mathrm{~cm}^{-1}$ and $1619.1 \mathrm{~cm}^{-1}$, whereas; $1375.3 \mathrm{~cm}^{-1}$ and $1379.1 \mathrm{~cm}^{-1}$ peaks confirmed the presence of $\mathrm{C}-\mathrm{H}$ deformations. PAM asymmetric stretching based vibrations were located at $2921.1 \mathrm{~cm}^{-1}$ and 2916.4 $\mathrm{cm}^{-1}$ for $\mathrm{C}-\mathrm{H}$ [19]. Additional peaks for graphene oxide were found to be situated at $3369.7 \mathrm{~cm}^{-1}$ and $3288.6 \mathrm{~cm}^{-1}$ for $\mathrm{OH}$ groups, whereas; $1649.2 \mathrm{~cm}^{-1}$ and $1655.0 \mathrm{~cm}^{-1}$ for the carbonyl group in the CS-PAMGO IR spectra [21].

After adsorption of Congo red dye, various new functional groups were discovered including $\mathrm{S}=\mathrm{O}$ stretching of sulphonic acid at 1130.0 $\mathrm{cm}^{-1}$ and $1120.3 \mathrm{~cm}^{-1}$, whereas; for characteristic aromatic skeletal vibrations were observed at $893.0 \mathrm{~cm}^{-1}$ and $823.2 \mathrm{~cm}^{-1}$. The peaks of C$\mathrm{C}$ bending vibrations and $\mathrm{C}-\mathrm{H}$ stretching vibrations of disubsituted aromatic compound were located at $619.3 \mathrm{~cm}^{-1}$ and $679.2 \mathrm{~cm}^{-1}$. A few peaks either shifted to lower or higher wave number and a few became more or less intense as observed comparatively with the spectra of control samples. $\mathrm{OH}$ and $-\mathrm{NH}$ stretching vibrations based peaks became less intense, $\mathrm{C}=\mathrm{C}$ stretching vibrations became more intense, $\mathrm{C}=\mathrm{O}$ of carbonyl shifted to lower wave number, and the peaks for $\mathrm{S}=\mathrm{O}$ stretching vibrations shifted towards lower wavenumber $[25,26]$. This confirms the participation of reactive functional groups in bonding after adsorption.

SEM results: Scanning electron microscope photographic results of hydrogels were acquired and analyzed to observe the presence of any porosity, channels, and cross-linkage networks. Figure 4 shows interesting comparative results between CS-PAM hydrogels and CSPAM-GO hydrogels. In Figure 4 IA CS-PAM hydrogels seem to have a well cross-linked channel-based network at 500X magnifications with porous structures. 
Citation: Sami AJ, Butt YN, Nasar S (2018) Elimination of a Carcinogenic Anionic Dye Congo Red from Water Using Hydrogels Based on Chitosan, Acrylamide and Graphene Oxide. J Bioprocess Biotech 8: 334. doi:10.4172/2155-9821.1000334

I.

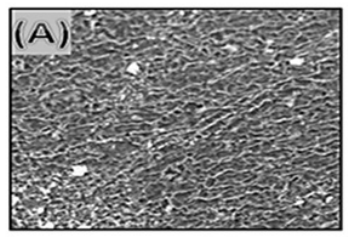

II.
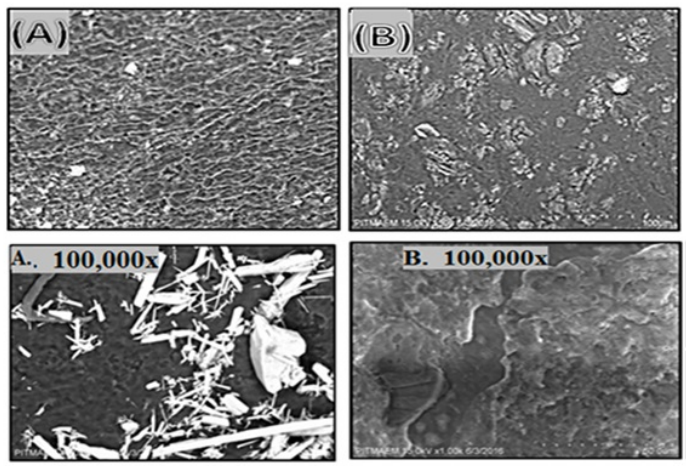

III.
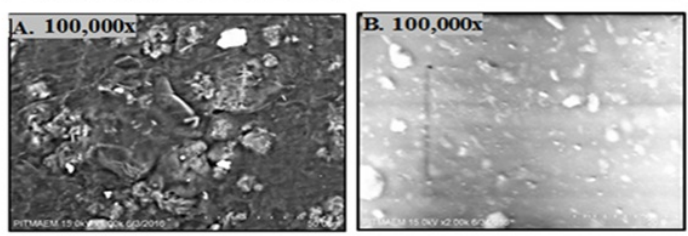

Figure 4: (I) (a) CS-PAM (b) CS-PAM-GO; 4(II) CS-PAM hydrogel before and after adsorption; 4(III) CS-PAM-GO hydrogel before and after adsorption.

The overall structure of this hydrogel comprises of uniformity of channels and pores based morphology which leads to high swelling and adsorption efficacies [17]. There are ellipsoidal repeating structures in cross-linked structure with pores within the range of 40 to $80 \mu \mathrm{m}$ as visualized through SEM images scale. These are white colored structures which represent interlinkage agglomeration of CS with PAM. These accumulated structures are randomly spread throughout the hydrogel.

In case of CS-PAM-GO hydrogels (Figure 5), the cross-linkages with uniform network are masked due to the presence of additional component which is graphene oxide layers. Not only GO based hydrogels have restricted pore size but also rough and uneven surface with ridges and elevated marks. The images are magnified to 500X where pores are observed yet channels are not. In the background, the network of PAM with CS is still visible in darker part.
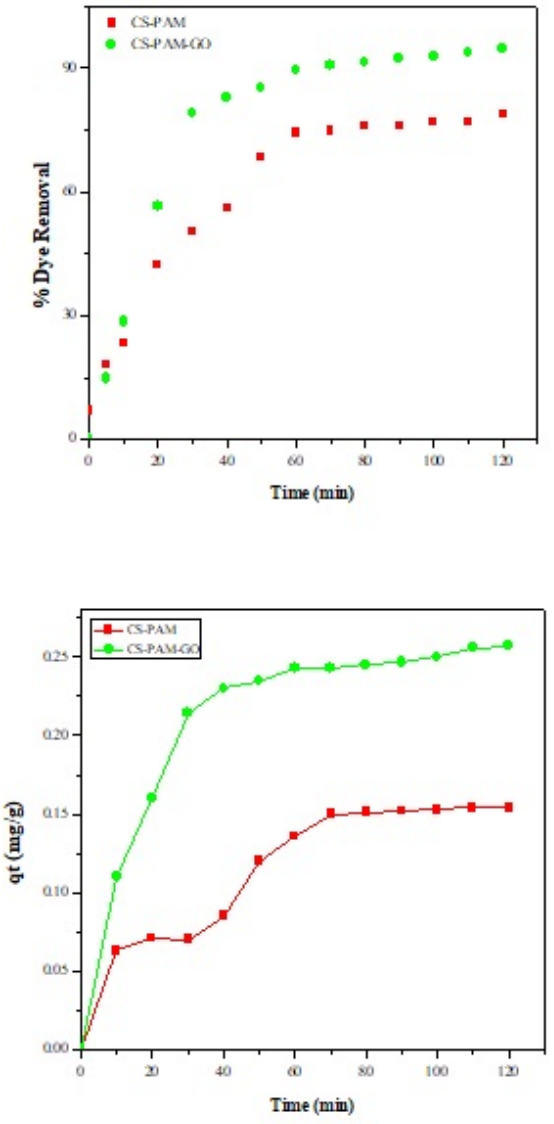

Figure 5: Percentage Congo red Dye removal by hydrogels (A) (left). The adsorption kinetics based graph qt vs $\mathrm{t}$ (B) (right).

The overall structure of graphene oxide containing hydrogel has irregular and coarse surface where additional layers of graphene oxide have covered the channels seen in the CS-PAM hydrogels.

The SEM results of hydrogels before and after Congo red dye removal were obtained to determine the topographical details. Both hydrogels and hydrogels, with and without graphene oxide had agglomerated structures of CS with PAM which were also linked to GO nanosheets in CS-PAM-GO hydrogels. Figure 4 II A shows CS-PAM hydrogels magnified to $100,000 \mathrm{X}$ where it appears quite coarse before the adsorption of Congo red dye. It becomes smooth and plain after adsorption of dye as shown in Figure 4 II B. In addition to these details offered by CS-AM hydrogels with adsorbed Congo red dye, a cleft like appearance is seen at the focused part of hydrogel containing smooth pore like channels with adsorbed Congo red dye. The appearance of Congo red dye on hydrogels is uniform yet bloated.

In Figure 4 IIIA, the CS-PAM-GO hydrogels without dye adsorption are found to have uneven and irregular surface with visibility of minor pores at magnification 100,000X. On adsorbing Congo red dye (Figure 5), these hydrogels seem to have very smooth surface due to adherent dye molecules which cover the surface. SEM results are confirmatory results which justify the mechanism and adsorption of dye on to the hydrogels. Dark areas specify the pores available in the hydrogel which get masked due to the adsorption of Congo red dye. 
Page 7 of 11

Anti-microbial activity: Anti-microbial activity of hydrogels checked against E. coli strain JM-109 produced zones of inhibition in test plates. Inhibition zone was observed for both the hydrogels with and without graphene oxide. However, hydrogels containing graphene oxide had larger zone than those without graphene oxide due to oxidative mechanism. This peripheral zone around the hydrogels matched the negative plate showing anti-microbial activity while remaining part matched positive control plate comprised of bacterial culture. The mechanism proposes that superoxide anion is generated by graphene oxide which disrupts the bacterial membrane. The graphene oxide nano-sheets have functional groups containing oxygen which trap the bacteria into the nano-sheets. When bacteria come in direct contact with these nano-sheets, the sharp edges of graphene oxide nano walls disrupt the cellular membranes through oxidative stress.

Folding endurance: The graphene oxide nanoparticles increased folding endurance of hydrogels by offering higher flexibility, endurance, and strength. The folding endurances for CS-PAM and CSPAM-GO hydrogels were satisfactory yet greater for the later. This confirmed that hydrogels can maintain the integrity while being used as agents for dye removal without any breakage.

Adsorption and kinetics: The adsorption efficiency of hydrogel was monitored over the time duration of 120 minutes working solutions of $0.1 \%$ CR dye (5x). Figure 4 represents the absorption spectra for CSPAM and CS-PAM-GO hydrogels. The graph plot had a similar trend for both the hydrogels with gradual increase in percentage dye removal until it reached equilibrium where no more dye was adsorbed by the hydrogels. CS-PAM hydrogel in $5 \times \mathrm{CR}$ dye solution resulted in $79 \%$ adsorption before attaining equilibrium. Similarly, CS-PAM-GO hydrogel adsorbed $94.7 \%$ CR dye before reaching equilibrium.

Adsorption of Congo red dye depends on the availability of reactive functional groups on the hydrogels. Scheme 1 shows the schematic representation of linkage between chitosan and Congo red dye where ammonium group of Chitosan links to the sulphonyl of the Congo red dye.

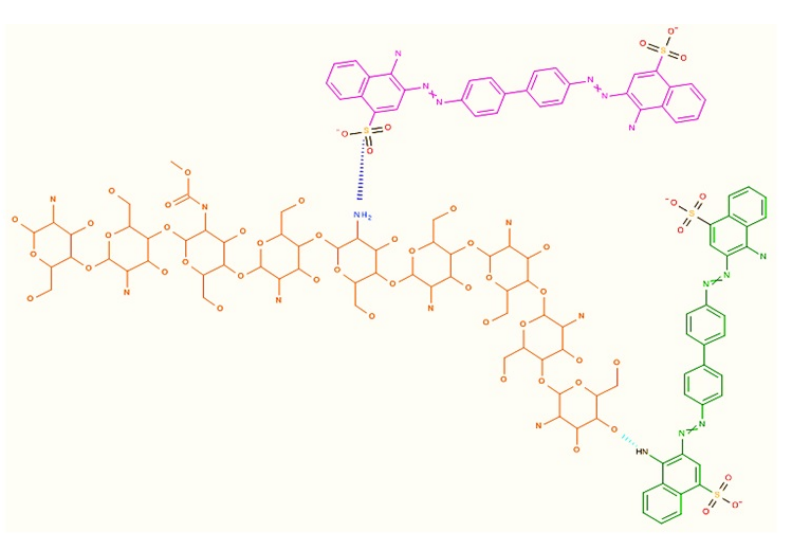

Scheme 1: Linkages of Chitosan with Congo red.
The linkage between chitosan and Congo red dye is also based on electrostatic nature. Hydrogen bonding between amino group from the dye and hydroxyl group from chitosan has also been demonstrated. Together these components produce a porous network where dye molecules incorporate for adsorption. CS-PAM-GO hydrogels had greater stability as compared to CS-PAM hydrogels and the adsorption efficiency of these hydrogels was greater than that of CS-PAM due to the enhanced surface area and intercalation of GO in the hydrogels. Furthermore, usage of graphene oxide in hydrogels offered greater compressive strength.

Adsorption kinetics was studied to determine the mechanism and efficiency of hydrogels for dye adsorption. The values of qt in $\mathrm{mg} / \mathrm{g}$ were calculated and graph was plotted as shown in Figure 4B. The maximum value of qt for CS-PAM hydrogels was observed to be 0.15 $\mathrm{mg} \mathrm{g}^{-1}\left(2.1 \times 10^{-4} \mathrm{~mol} \mathrm{~g}^{-1}\right)$ while CS-PAM-GO had maximum value of $0.25 \mathrm{mg} \mathrm{g}^{-1}\left(3.5 \times 10^{-4} \mathrm{~mol} \mathrm{~g}^{-1}\right)$. The values of dye adsorption were supplemented to the graphs for determination of order of reaction followed by the hydrogels.

Table 2 shows the calculated values of pseudo first order and pseudo second order kinetics. Figure $6 \mathrm{~A}$ shows that the values of regression for both the hydrogels with and without graphene oxide are very low in case of pseudo first order. The qe values did not correspond well in it, therefore; hydrogels did not follow first order of reaction. However, the adsorption values fit well for pseudo second order reaction for both types of hydrogels as shown in Figure 6B.
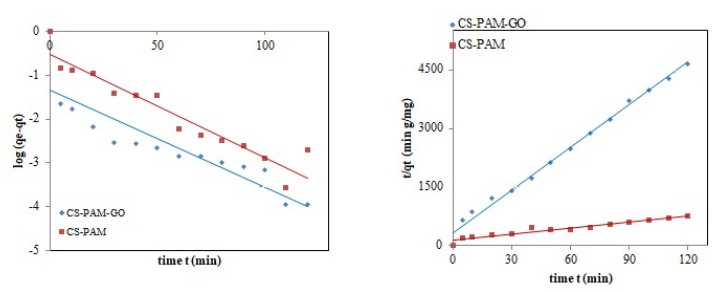

Figure 6: (A) Pseudo 1st order Kinetics for Congo red dye adsorption at different concentrations of solution (left); (B) Pseudo 2nd order Kinetics for Congo red dye adsorption at different concentrations of solution (right).

In case of pseudo second order of reaction there is close agreement between $\mathrm{q}_{\mathrm{e}}$ values with good regression and excellent linearity. The value of qe for CS-PAM hydrogels was 0.97 while it was optimum for CS-PAM-GO with regression value 0.99 . The adsorption mechanism explains different stages involved in the transport of adsorbate molecules from liquid phase to the solid phase including entrance into the interior of pores on the adsorbent surface. The overall process of adsorption is either controlled by one or more factors including pore size, film diffusion, external diffusion, and surface diffusion. Similar work has also been reported by Dawood and Sen [24].

\begin{tabular}{|l|l|l|l|l|l|l|l|l|}
\hline Hydrogel & qe exp 1/slope & qe (cal) & K1 & R1 & K2 & h & R2 & qe (calc) \\
\hline CS-PAM & 5.24 & 0.19 & 0.46 & 84 & 139.6 & $3.7 \times 10^{3}$ & 93 & 5.18 \\
\hline
\end{tabular}


Citation: Sami AJ, Butt YN, Nasar S (2018) Elimination of a Carcinogenic Anionic Dye Congo Red from Water Using Hydrogels Based on Chitosan, Acrylamide and Graphene Oxide. J Bioprocess Biotech 8: 334. doi:10.4172/2155-9821.1000334

Page 8 of 11

\begin{tabular}{|l|l|l|l|l|l|l|l|l|}
\hline CS-PAM-GO & 36.4 & 0.02 & 0.8 & 70 & 341.2 & $4.4 \times 10^{5}$ & 99 & 36.3 \\
\hline
\end{tabular}

Table 2: Adsorption kinetics and Calculations.

Conditions provided to the system. For this process designing and proper control of adsorption system it becomes necessary to determine the underlying mechanism to reach dynamic behavior of system. Efficient removal of Congo red dye through quick adsorption on hydrogel was determined to be rapid at initial time of contact for the change in solid and liquid system based on sorption. The transfer of solute dye molecules was dependent on the external mass transfer including intra particle diffusion, boundary layer diffusion, or even both these mechanisms.

Adsorption usually occurs in four steps. Migration of dye molecules present in the bulk solution to the adsorption surface and then diffusion from the boundary layer to the exterior surface of the hydrogels, then to the sites of adsorption and finally intra particle diffusion which is interior of the hydrogels based on pores and channels created by polymers. The control step is distributed often between intra particle diffusion and the external mechanism of transport. Sorption is controlled by diffusion which takes place at the earlier stages and adsorbent molecules get highly loaded with dye molecules. The diffusion into pores is controlled by the intra particle diffusion.

The plot shown in Figure 7 with values mentioned in Table 3 depicts division of time range into linear regions with multiple adsorption stages for CS-PAM and CS-PAM-GO hydrogels.
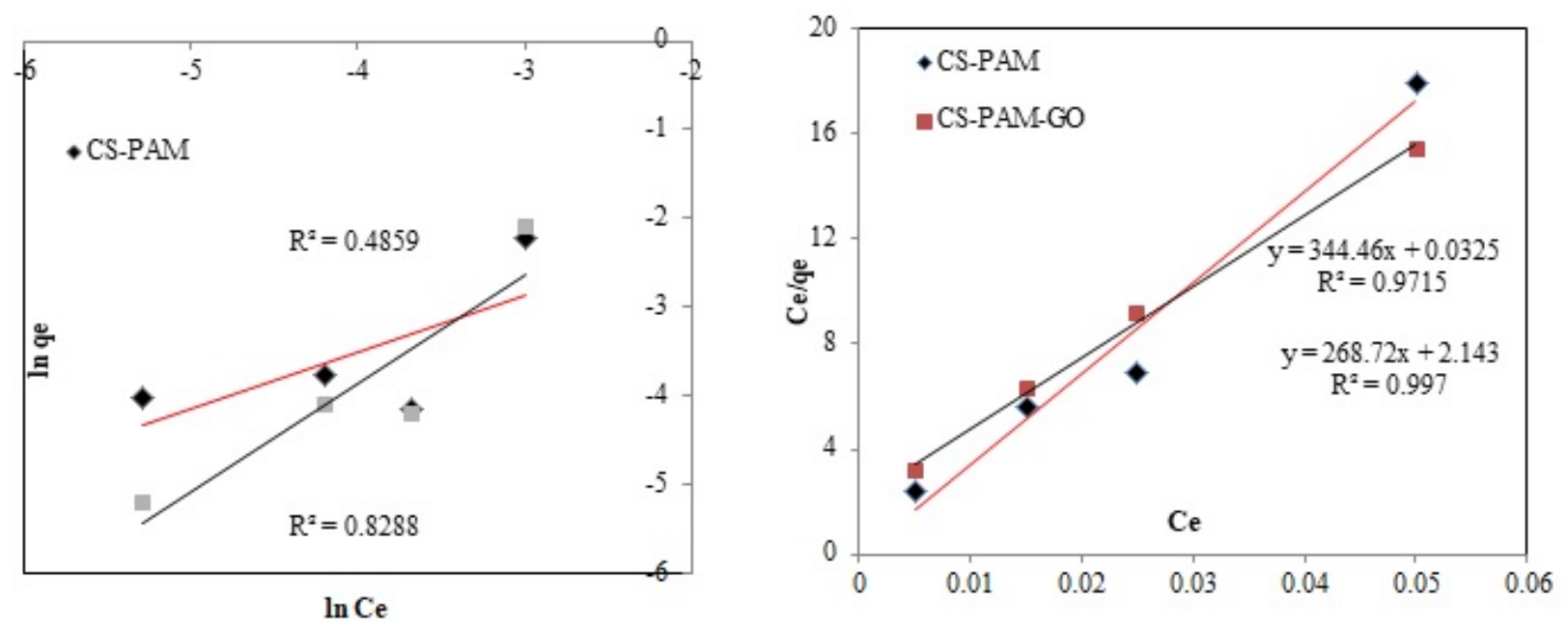

Figure 7: (A) Freundlich Isotherm plot (left); (B) Langmuir Isotherm plot (right).

For both hydrogels, there are two regions specified using the plot including external mass transfer followed by the intra particle diffusion which signifies that dye ions were transported to external surface of adsorbent particle via film diffusion in a rapid manner.
On entering the pores, the diffusion mechanism turned to intra particle diffusion however intra particle diffusion is not the only rate controlling factor here. Other mechanisms including complexation and the exchange of ions are also found to be involved.

\begin{tabular}{|l|l|l|l|}
\hline Conc. & qt & $\mathbf{t}$ & $\mathbf{t} 0.5$ \\
\hline CS-PAM & 0.0986 & 30 & 5.47 \\
\hline & 0.1457 & 60 & 7.74 \\
\hline CS-PAM-GO & 0.1543 & 120 & 10.95 \\
\hline & 0.0214 & 30 & 5.47 \\
\hline & 0.0243 & 60 & 7.74 \\
\hline
\end{tabular}

Table 3: Intra particle diffusion. 
Page 9 of 11

The Adsorption isotherms including Langmuir and Freundlich isotherms were also plotted to observe the possibility of interaction present between the adsorbate species. These sets of assumptions are mainly relevant to the homogeneity and heterogeneity of the adsorbents and the type of coverage they do. The adsorption data was described by Freundlich and Langmuir Isotherms as shown in Figures $8 \mathrm{~A}$ and $8 \mathrm{~B}$.

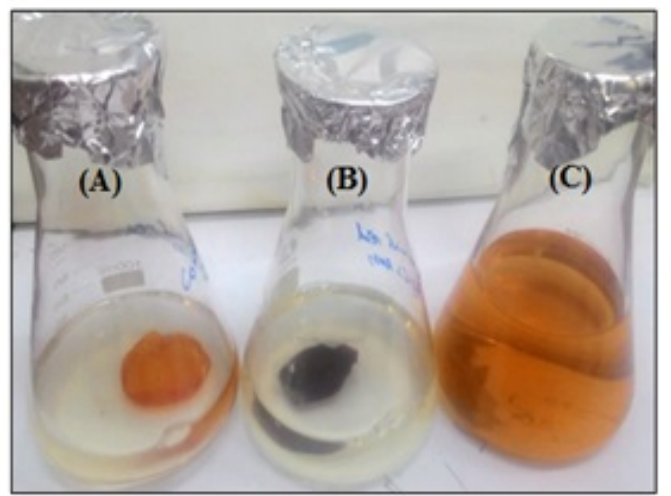

Figure 8: (a) Commerical wastewater dye adsorption by chitosan polyacrylamide hydrogel; (b) chitosan polyacrylamide graphene oxide nanocomposite alloy; (c) with control.

Freundlich isotherm is empirical relationship which describes the adsorption of solute particles present in a liquid to the solid surface of the adsorbent assuming that different energies are involved for different sites of the heterogeneous surface. The experimental adsorption data of the hydrogels was plotted for $\ln \mathrm{q}_{\mathrm{e}}$ and $\ln \mathrm{Ce}$ of Freundlich which demonstrated low linearity with less regression value. However, the experimental data for adsorption of hydrogels followed Langmuir isotherm based mechanism with acceptable regression value of 0.97 and 0.99 . This mechanism is based on the assumption that maximum adsorption takes place when saturated monolayer of solute molecules exists on the adsorbent where energy of adsorption remains constant and adsorbate molecules do not migrate in the surface plane [15].

\section{Application}

Dye removal from commercial wastewater sample: The real time application of dye removal from textile wastewater sample was attempted by obtaining commercial wastewater sample from a local vendor. Similar parameters including percentage dye removal and adsorption kinetics were studied to determine the potential of hydrogel in actual textile wastewater sample for application. Maximum dye adsorption by the CS-PAM control and CS-PAM-GO test hydrogels was observed in reactive red dye containing commercial wastewater sample with $85 \%$ and $89 \%$ of dye removal by the respective hydrogels. Both hydrogels followed pseudo second order reaction with interrelated qe values of 37.2 and 39.8 at low discerning concentration while 8.3 and 9.6 at high concentration subsequently supporting Langmuir isotherm (Table 4). The adsorption kinetics explains that initially adsorption is high, and it gradually subsides when dye molecules begin occupying the pores until it reaches equilibrium where no more dye molecules are adsorbed. There's lack of available sites at equilibrium.

\begin{tabular}{|c|c|c|c|}
\hline Conc. & $q t$ & $\mathbf{t}$ & to.5 \\
\hline \multirow{3}{*}{$1 x$} & 0.021428 & 30 & 5.47 \\
\hline & 0.024285 & 60 & 7.74 \\
\hline & 0.025714 & 120 & 10.95 \\
\hline \multirow{3}{*}{$3 x$} & 0.018571 & 30 & 5.47 \\
\hline & 0.032857 & 60 & 7.74 \\
\hline & 0.032857 & 120 & 10.95 \\
\hline \multirow{3}{*}{$5 x$} & 0.015714 & 30 & 5.47 \\
\hline & 0.018571 & 60 & 7.74 \\
\hline & 0.022857 & 120 & 10.95 \\
\hline \multirow{3}{*}{$10 x$} & 0.098571 & 30 & 5.47 \\
\hline & 0.145714 & 60 & 7.74 \\
\hline & 0.154285 & 120 & 10.95 \\
\hline
\end{tabular}

Table 4: Table shows the kinetics of commercial dye removal by novel hysrogel.

In the initial concentration of sample, this highlights that at low initial dye concentration, amount of anionic dye is more in comparison. Initial concentration of dye offers driving force to overcome resistance against mass transfer of the dye molecules between solid and liquid phase transition. At fixed dose of adsorbent, adsorption capacity increases with an increase of dye solution concentration. Similarly, at low concentration solution, ratio of initial amount of dye molecules to the availability of adsorption sites is low.

The anticipated constituents present in the textile sample include chloride ions from bleaching chemicals, dissolved carbon compounds, 
and surfactants which are removed through chemical and biological methods. Textile effluents have high COD and BOD, toxic compounds, suspended solids, and carcinogenic dyes. Since dyes are not degradable, therefore they may only be removed through the most cost-effective adsorption method. The commercial sample contained reactive red dye which is another recalcitrant organic sodium salt with similar $\mathrm{NH}_{2}$ and $\mathrm{SO}_{3}$ functional groups available for adsorption as that of the Congo red. Intake of reactive red dye causes potential chronic disorders and cancer. The reactive red dyes are also anionic in nature and hydrolyse in aqueous solution therefore binds covalently to the textiles. These dyes are photolytically and chemically stable, so they persist in environment and their aromatic moiety carries sulfonic groups. Textile processing wastewater usually has $10-200 \mathrm{mg} / \mathrm{l}$ dye content which leaves high coloured discharge with aesthetic problems. The dye also presents ecotoxic hazard introducing potential bioaccumulation dander affecting humans by transport through food chain. Among constituents, CS-PAM-GO is also expected to show adsorption towards heavy metals, cationic and other anionic dyes due to availability of these functional groups. This research paves the path for future studies on similar hydrogels to test the adsorption potential of dye and removal of other harmful constituents from the textile wastewater simultaneously

\section{Discussion}

Novel hydrogels based on chitosan polyacrylamide prepared with and without graphene oxide at $55^{\circ} \mathrm{C}$ were optimized for efficient removal of Congo red and commercial dyes from commercial wastewater sample. Initially, rate of adsorption was slow for hydrogels immersed in dye solution however; it then increased to a log phase with high adsorption of Congo red dye. Reason behind this radical change was the initial time required for dye molecules to overcome resistance for the mass transfer between aqueous phase and solid phase. There was instantaneous utilization of active sites present in the hydrogels which offered bulk diffusion and a wide adsorbent surface area for dye removal. By the log phase, dye molecules reached the macro-pores of adsorbent hydrogel through pore diffusion. It also followed the mechanism of intra-particle diffusion. There was continuous movement of dye matter from liquid to the adsorbent until last stage of equilibrium was attained. Swelling effect was also affected by dye molecules having sufficient energy to create a force on the surface of hydrogel. Higher concentration of dye solution increased the interaction between dye molecules and hydrogels.

SEM results have also substantiated the above regard by demonstrating surface topography of the hydrogels having a fine and uniform crosslinking based network of chitosan chains interlinked with polyacrylamide network. In addition to this, graphene oxide occupies the pores and channels in a hydrogel enhancing the surface area for adsorption. One of the factors for an increased adsorption capacity is the uneven surface having pores as observed at a particularly higher magnification. After adsorption all the cave like structures are found to be covered with a layer of dye molecules throughout the hydrogels. This turns the surface to become smooth again covered with dye molecules.

Swelling analysis of hydrogels is a true hyperbolic curve with rapid increase of water molecules to penetrate the hydrogel due to fine, even, and uniform porous structure of these hydrogels. It is easy for water molecules to reach and settle in the cross-linked network created by chitosan and polyacrylamide three dimensional structure but again it takes certain time in minutes for dye molecules to reach the channels in hydrogels.

According to the given values, hydrogels followed second order of reaction due to greater effective regression values (closer to unity). The graphs showed straight (linear) lines which corresponded to mechanism of several stages. Adsorption of dye through hydrogel has to pass stages involving mass transport from aqueous medium to the solid medium and diffusion into channels and pores present in the interior of hydrogel. This makes this process a bit slower though. The experimental values of amount of dye adsorbed $\mathrm{q}_{\mathrm{e}}(\exp )$ at equilibrium were similar to the calculated values of dye adsorbed qe (calc) for the pseudo second order rate kinetics. This also suggests that the adsorption of dye on to hydrogels involves behavior of chemisorption with a lot of sharing and exchange of electrons present between anionic groups of the dye molecules and the cationic groups of the hydrogels. It is in chemisorption or chemical adsorption that the molecules of dyes adhere to the adsorbent through chemical linkage such as covalent bonding.

\section{Conclusion}

Congo red is an anionic dye used in textile industries presented as a carcinogenic contaminant in the wastewater released from the textile mills and tanneries. Researchers are in search of economically efficient and convenient methods for the removal of Congo red from the industrial wastewater such as adsorption. This study was conducted; aiming the application of hydrogels based on chitosan/polyacrylamide composite and Graphene oxide (GO) for wastewater purification system. Graphene was prepared from used battery cells and used as starting material for GO. Results imply that under standard conditions, $200 \mathrm{mg}$ of Congo red was removed by one gram of chitosan present in the hydrogels. A comparative study of hydrogels with and without GO was also carried out. Earlier different workers have used chitosan beads for the removal of Congo red for wastewater treatment, however; preparation and extraction of used beads after treatment can be laborious. The use of hydrogels has an advantage over this method as stable hydrogels can be removed conveniently. Chitosan polyacrylamide hydrogels adsorb Congo red dye efficiently due to the presence of acrylamide and chitosan which offer hydrophilic functional groups. These hydrogels have greater swelling leading to greater adsorption. Addition of graphene oxide to the hydrogel improves the properties of the hydrogels required during the process of purification. This shows that the use of hydrogels is economical and efficient in removing dyes from the industrial water waste, under mild conditions.

\section{References}

1. Aziz MA, Cabral JD, Brooks HJ, Moratti SC, Hanton LR (2012) Antimicrobial properties of a chitosan dextran-based hydrogel for surgical use. Antimicrob Agents 56: 280-287.

2. Banisharif A, Hakim Elahi S, Anaraki Firooz A, Khodadadi A, Mortazavi Y (2013) TiO2/Fe3O4 nanocomposite photocatalysts for enhanced photodecolorization of Congo red dye. Int J Nanosci Nanotech 9: 193-202.

3. Berger J, Reist M, Mayer JM, Felt O, Peppas N, et al. (2004) Structure and interactions in covalently and ionically crosslinked chitosan hydrogels for biomedical applications. Eur J Pharm Biopharm 57: 19-34.

4. Bhalkaran S, Wilson LD (2016) Investigation of Self-Assembly Processes for Chitosan-Based Coagulant-Flocculant Systems: A Mini-Review. Int J Mol Sci 17: 1662.

5. Bisschops I, Spanjers H (2003) Literature review on textile wastewater characterization. Environm Tech 24: 1399-1411. 
Citation: Sami AJ, Butt YN, Nasar S (2018) Elimination of a Carcinogenic Anionic Dye Congo Red from Water Using Hydrogels Based on Chitosan, Acrylamide and Graphene Oxide. J Bioprocess Biotech 8: 334. doi:10.4172/2155-9821.1000334

Page 11 of 11

6. Chen SC, Wu YC, Mi FL, Lin YH, Yu LC, et al. (2004) A novel pHsensitive hydrogel composed of $\mathrm{N}$, O-carboxymethyl chitosan and alginate cross-linked by genipin for protein drug delivery. J Controlled Release 96: 285-300.

7. Dawood S, Sen TK (2012) Removal of anionic dye Congo red from aqueous solution by raw pine and acid-treated pine cone powder as adsorbent: equilibrium, thermodynamic, kinetics, mechanism and process design. Water Res 46: 1933-1946.

8. Domingues JA, Bonelli N, Giorgi R, Fratini E, Gorel F, et al. (2013) Innovative hydrogels based on semi-interpenetrating $\mathrm{p}$ (HEMA)/PVP networks for the cleaning of water-sensitive cultural heritage artifacts. Langmuir 29: 2746-2755.

9. Dragan ES (2014) Design and applications of interpenetrating polymer network hydrogels. A review. Chem Eng J 243: 572-590.

10. Gad Y (2008) Preparation and characterization of poly (2-acrylamido-2methylpropane-sulfonic acid)/Chitosan hydrogel using gamma irradiation and its application in wastewater treatment. Radiation Phy Chem 77: 1101-1107.

11. Heidari F, Razavi M, Bahrololoom ME, Bazargan-Lari R, Vashaee D, et al. (2016) Mechanical properties of natural chitosan/hydroxyapatite/ magnetite nanocomposites for tissue engineering applications. Mater Sci Eng 65: 338-344.

12. Hou H, Zhou R, Wu P, Wu L (2012) Removal of Congo red dye from aqueous solution with hydroxyapatite/chitosan composite. Chem Eng J 211: 336-342.

13. Kumar PS, Kirthika K (2009) Equilibrium and kinetic study of adsorption of nickel from aqueous solution onto bael tree leaf powder. J Eng Sci Tech 4: 351-363.

14. Liu C, Bai R (2014) Recent advances in chitosan and its derivatives as adsorbents for removal of pollutants from water and wastewater. Current Opinion Chem Eng 4: 62-70.

15. Leme DM, Oliveira GARD, Meireles G, Brito LB, Rodrigues LDB, et al. (2015) Eco-and genotoxicological assessments of two reactive textile dyes. J Toxico Environm Health 78: 287-300.

16. Mahmood R, Javaria I (2014) Adsorption of Commercial Dye (Red-ClsBar) Onto Ash Collected from Brick Kiln. World Applied Sci J 29: 968-977.
17. Martinez-Ruvalcaba A, Sanchez-Diaz J, Becerra F, Cruz-Barba L, Gonzalez-Alvarez A (2009) Swelling characterization and drug delivery kinetics of polyacrylamide-co-itaconic acid/chitosan hydrogels. Express Polym Lett 3: 25-32.

18. Mochalova A, Zaborshchikova N, Knyazev A, Smirnova L, Izvozchikova $\mathrm{V}$, et al. (2006) Graft polymerization of acrylamide on chitosan: Copolymer structure and properties. Polymer Sci Series A 48: 918-923.

19. Panic VV, Velickovic SJ (2014) Removal of model cationic dye by adsorption onto poly (methacrylic acid)/zeolite hydrogel composites: kinetics, equilibrium study and image analysis. Sep Purif Technol 122: 384-394.

20. Perju M, Dragan E (2010) Removal of azo dyes from aqueous solutions using chitosan based composite hydrogels. Ion Exchange Lett 3: 7-11.

21. Qiu X, Hu S (2013) "Smart" materials based on cellulose: a review of the preparations, properties, and applications. Materials 6: 738-781.

22. Shahriary L, Athawale AA (2014) Graphene oxide synthesized by using modified hummers approach. Int J Renew Energy Environ Eng 2: 58-63.

23. Sun JY, Zhao X, Illeperuma WR, Chaudhuri O, Oh KH, et al. (2012) Highly stretchable and tough hydrogels. Nature 489: 133-136.

24. Tan I, Ahmad A, Hameed B (2009) Adsorption isotherms, kinetics, thermodynamics and desorption studies of 2, 4, 6-trichlorophenol on oil palm empty fruit bunch-based activated carbon. J Hazard Mater 164: 473-482.

25. Vakili M, Rafatullah M, Salamatinia B, Abdullah AZ, Ibrahim MH, et al. (2014) Application of chitosan and its derivatives as adsorbents for dye removal from water and wastewater: A review. Carbohydr Polym 113: 115-130.

26. Van de Velde K, Kiekens P (2004) Structure analysis and degree of substitution of chitin, chitosan and dibutyrylchitin by FT-IR spectroscopy and solid state 13 C NMR. Carbohydr Polym 58: 409-416.

27. Zhang N, Li R, Zhang L, Chen H, Wang W, et al. (2011) Actuator materials based on graphene oxide/polyacrylamide composite hydrogels prepared by in situ polymerization. Soft Matter 7: 7231-7239. 